\title{
Internal fixation combined with bone grafting for large intraosseous calcaneal lipoma: A case report
}

\author{
YONGXING CAO \\ Department of Orthopedics, Ruijin Hospital North, Medical School of Shanghai \\ Jiao Tong University, Shanghai 201800, P.R. China
}

Received May 31, 2017; Accepted September 11, 2017

DOI: $10.3892 / \mathrm{mco} .2017 .1421$

\begin{abstract}
Intraosseous lipoma is a rare benign bone tumor that has been reported to occur in the calcaneus and long bones. The etiology of intraosseous lipoma is unknown, although several theories have been proposed. The majority of the cases of intraosseous calcaneal lipoma reported in the literature were localized in the anterior portion of the calcaneus and were treated by curettage and bone grafting. However, for larger lipomas, no specific treatment protocol has been developed to date. We herein present a rare case involving a large intraosseous lipoma of the calcaneus in a 36-year-old man following hindfoot trauma. The lesion was treated by decortication followed by bone grafting and internal fixation, a surgical approach that, to the best of our knowledge, has not been previously described in the literature.
\end{abstract}

\section{Introduction}

Intraosseous calcaneal lipoma was first described in 1976 (1). This type of lesion is a rare benign bone tumor that is commonly asymptomatic, and is usually incidentally discovered on radiographs performed for unrelated disorders. Intraosseous calcaneal lipoma remains a poorly characterized tumor for orthopedic surgeons, and its etiology is completely unknown $(2,3)$. The indications for surgery and optimal surgical modalities remain controversial. We herein report a case of a large intraosseous lipoma of the calcaneus in a middle-aged man following a hindfoot injury.

\section{Case report}

A 36-year-old male construction worker visited Ruijin Hospital North (Shanghai, China) in November 2014, with a complaint

Correspondence to: Dr Yongxing Cao, Department of Orthopedics, Ruijin Hospital North, Medical School of Shanghai Jiao Tong University, 999 Xiwang Road, Jiading, Shanghai 201800, P.R. China

E-mail: caoyongxing@foxmail.com

Key words: bone tumor, calcaneus, internal fixation, lipoma, surgery of dull pain in his left heel over the last 5 months. The patient reported spraining his hindfoot 5 months ago and visiting a local hospital immediately after the injury. Plain-film radiographs were normal, and the emergency physician initially managed the sprain with rest and analgesics. However, the pain increased gradually over the next 5 months and the patient visited our Orthopedics Department.

Physical examination revealed mild tenderness in the left heel, without evidence of a palpable mass or soft tissue edema. The range of motion of both ankles and subtalar joints were within the normal range. The laboratory blood tests and urine analysis results were all normal.

Plain-film radiographs revealed a large, well-defined lytic lesion sized $\sim 2.5 \times 3 \mathrm{~cm}$ in size (Fig. 1A). A magnetic resonance imaging (MRI) scan of the left foot revealed a large cyst in the central portion of the calcaneus. T1-weighted MRI revealed an intramedullary lesion with a signal intensity equal to that of subcutaneous fat. T2-weighted images also displayed an intramedullary lesion with a hyperintense signal (Fig. 1B). On the basis of the clinical and typical diagnostic imaging findings, a diagnosis of intraosseous calcaneal lipoma was made, and the patient subsequently underwent surgery.

Surgery was performed under general anesthesia, with the patient placed in the lateral position. Using a lateral approach, the lateral wall of the calcaneus was exposed. When the cyst was decorticated, curettage of the intraosseous lipoma was thoroughly performed and the defect was filled with an artificial bone substitute. Due to the large bone defect and fragile cortical bone after curettage, an anatomically shaped plate was fixed to the lateral wall of the calcaneus after grafting (Fig. 1C). The entire specimen was sent to the Pathology Department for histological diagnosis. The wound was closed in anatomical layers, and the foot was dressed with pressure. The postoperative pathological findings included hyperplasia of adipose cells and blood vessels, with medullary trabecular bone. Intraosseous calcaneal lipoma was therefore confirmed (Fig. 2).

The heel pain improved immediately after the surgery, and the wound healed successfully. At 14 months after surgery, radiographs revealed that the lesion had healed well. The internal fixation was surgically removed (Fig. 1D). An MRI performed at that time displayed partial osseous integration of the calcium phosphate bone graft substitute, without evidence of tumor recurrence. 

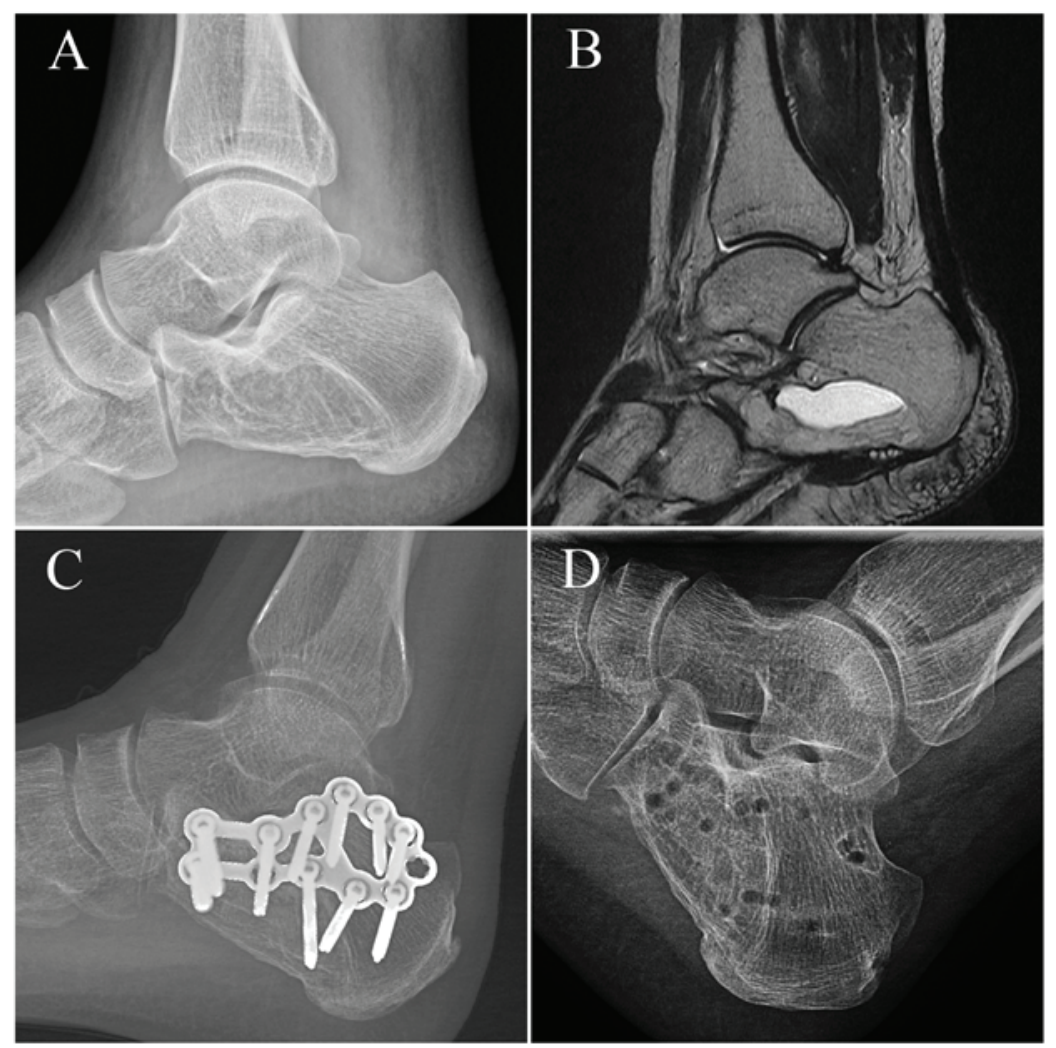

Figure 1. (A) Preoperative X-ray of the left heel showing the presence of an intraosseous lipoma. (B) Sagittal T2-weighted image showing a lesion in the left calcaneus with high signal intensity. (C) Postoperative X-ray of the left heel showing good internal fixation with bone grafting. (D) X-ray after internal fixation removal showing good bone healing.

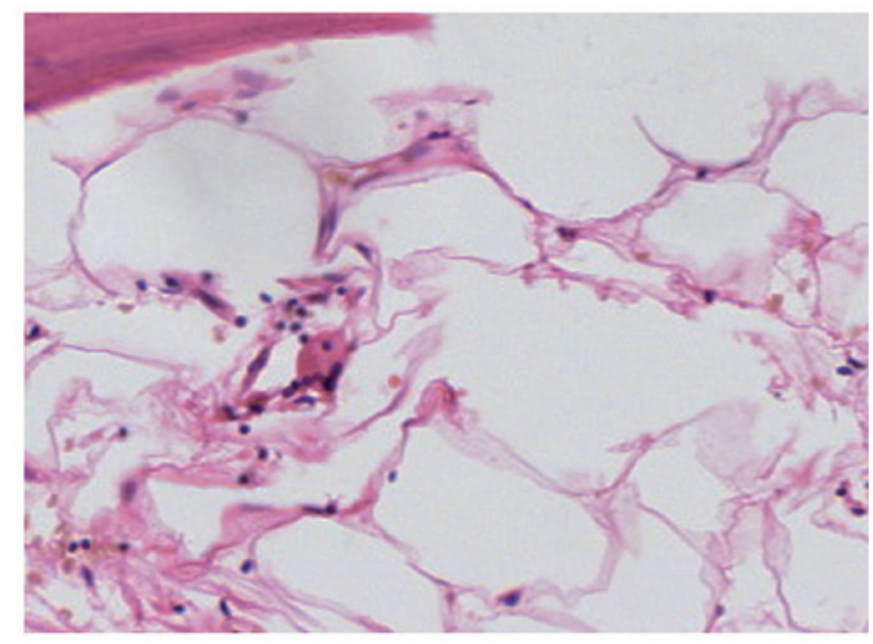

Figure 2. Histological specimen showing viable lipocytes and bony trabeculae (hematoxylin and eosin staining; magnification, $\mathrm{x} 250$ ).

\section{Discussion}

Intraosseous lipoma is a benign bone tumor that is rather uncommon, despite the abundance of adipose connective tissue in the bone marrow (1). Patients with intraosseous lipomas are often asymptomatic, and several cases are incidentally discovered. The etiology of intraosseous calcaneal lipoma remains unclear, although several hypotheses have been proposed, including healing bone infarction and post-traumatic secondary bone reaction $(2,3)$. In the present study, the patient noticed increasing pain following a hindfoot trauma. It was therefore hypothesized that a traumatic lesion may be a predisposing factor in this case.

Intraosseous calcaneal lipoma is usually located between the anterior and middle third of the calcaneus, also referred to as the neutral triangle. This area is devoid of the trabecular network crossing the calcaneus (4). However, the lesion in the present case was larger compared with those previously reported. In addition, the lesion extended to the full breadth of the calcaneus lateromedially in the coronal plane, and occupied $\sim 75 \%$ of the anteroposterior length of the calcaneus.

Intraosseous calcaneal lipoma cannot be diagnosed using plain radiographs alone. If the radiographs show an osteolytic and well-circumscribed lesion with a thin sclerotic rim, this may be misdiagnosed as a simple bone cyst, aneurysmal bone cyst, giant cell tumor, or non-ossifying fibroma (5-7). Biopsy for histopathological analysis is optimal, but invasive (8). Recently, with high-quality imaging modalities such as computed tomography or MRI, intramedullary lipomas and other bone lesions may be diagnosed without the need for bone biopsy $(9,10)$. Identification of the fat component signal on MRI is diagnostic of an intraosseous lipoma on the basis of the high signal intensity on both T1- and T2-weighted sequences, and obvious signal reduction on fat suppression images $(10,11)$.

Asymptomatic small cysts in non-weight-bearing areas may be observed for progression, but the natural course remains unknown. The most appropriate treatment for intraosseous calcaneal lipoma is likely to depend on symptoms, location 
and size (12-14). Intraosseous calcaneal lipoma 'of critical size' has been defined as a lesion extending the full breadth of the calcaneus in the coronal plan, and occupying $\geq 30 \%$ of the anteroposterior length of the calcaneus (15).

Curettage and grafting is the standard surgical approach to the treatment of these benign lesions. The conventional treatment is curettage of the lesions with bone grafting, followed by external fixation in the ankle joint. However, in the present case, the intraosseous calcaneal lipoma was larger compared with those previously reported. Curettage of the lesion created a considerable bone defect, which reduces bone strength. The middle part of the calcaneus plays an important role in force transmission. Thus, internal fixation materials are crucial for the reconstruction of the anatomy.

In the present case, internal fixation combined with bone grafting after curettage of the lesion was proven to be an effective and safe treatment choice for the large interosseous calcaneal lipoma.

\section{References}

1. Poussa M and Holmström T: Intraosseous lipoma of the calcaneus. Report of a case and a short review of the literature. Acta Orthop Scand 47: 570-574, 1976.

2. Schatz SG, Dipaola JD, D'Agostino A, Hanna R and Quinn SF: Intraosseous lipoma of the calcaneus. J Foot Surg 31: 381-384, 1992.

3. Greenspan A, Raiszadeh K, Riley GM and Matthews D: Intraosseous lipoma of the calcaneus. Foot Ankle Int 18: 53-56, 1997.
4. Muramatsu K, Tominaga Y, Hashimoto $\mathrm{T}$ and Taguchi T: Symptomatic intraosseous lipoma in the calcaneus. Anticancer Res 34: 963-966, 2014.

5. Karthik K and Aarthi S: Intraosseous lipoma of the calcaneus mimicking plantar fascitis. Foot Ankle Surg 17: e25-e27, 2011.

6. Mandl P, Mester A and Balint PV: A black hole in a bone - intraosseous lipoma. J Rheumatol 36: 434-436, 2009.

7. Revenga Martínez M, Bachiller Corral FJ, Rubio García J, Muñoz Beltrán M and Zea Mendoza AC: Cystic lesion of the calcaneus. Intraosseous lipoma. Reumatol Clin 3: 139-142, 2007 (In Spanish).

8. Rose RE and Golding T: Intraosseous lipoma. Is a biopsy necessary? West Indian Med J 55: 291-292, 2006.

9. Ketyer S, Brownstein S and Cholankeril J: CT diagnosis of intraosseous lipoma of the calcaneus. J Comput Assist Tomogr 7: 546-547, 1983

10. Richardson AA, Erdmann BB, Beier-Hanratty S, Lautz D, Jacobs PM, Julsrud ME and Ringstrom JB: Magnetic resonance imagery of a calcaneal lipoma. J Am Podiatr Med Assoc 85: 493-496, 1995

11. Genchi V, Scialpi M, Scarciolla G, Dimauro F and Trigona A: Intraosseous lipoma of the calcaneus. Characterization with computerized tomography and magnetic resonance in a case. Radiol Med 99: 86-88, 2000 (In Italian)

12. Bagatur AE, Yalcinkaya M, Dogan A, Gur S, Mumcuoglu E and Albayrak M: Surgery is not always necessary in intraosseous lipoma. Orthopedics 33, 2010.

13. Neuber M, Heier J, Vordemvenne T and Schult M: [Surgical indications in intraosseous lipoma of the calcaneus. Case report and critical review of the literature]. Unfallchirurg 107: 59-63, 2004.

14. Bertram C, Popken F and Rütt J: Intraosseous lipoma of the calcaneus. Langenbecks Arch Surg 386: 313-317, 2001.

15. Pogoda P, Priemel M, Linhart W, Stork A, Adam G, Windolf J, Rueger JM and Amling M: Clinical relevance of calcaneal bone cysts: A study of 50 cysts in 47 patients. Clin Orthop Relat Res 424: 202-210, 2004 